\title{
Investigation of wooden sheet pile wall in an aggressive environment using non-destructive methods
}

\author{
Marek Wyjadłowski*, Tomasz Nowak and Anna Karolak \\ Wroclaw University of Technology, Wybrzeze Wyspianskiego 27, 50-370 Wroclaw, Poland
}

\begin{abstract}
The paper presents the study of the strength parameters of wood samples from the retaining structure, working for about 60 years in an aggressive sea water environment. Samples from the variable water level zone and situated in the watered ground were tested. Non-destructive examinations were performed using the following methods: the stress wave technique and the ultrasonic wave technique. The results presented below can lead to the conclusion that both methods: the stress wave by Fakopp Microsecond Timer device and the ultrasonic wave by Sylvatest Trio correspond with each other and result in similar outcomes. However, in order to obtain more detailed and comprehensive information on the elements properties, more research should be conducted.
\end{abstract}

\section{Introduction}

Wooden elements of the structure are subjected to various biological, chemical and atmospheric factors. These factors cause material destruction and reduce the durability and reliability of the structural system. In the case of important and responsible facilities, procedures for monitoring stresses, displacements to prevent failures are implemented (structural health monitoring). Non-destructive material testing is also used to determine variability of the strength parameters.

The research presented in the paper was made on wood samples from an unusual but responsible retaining structure of the port quay. The subject port quay can be described as a retaining construction with a cantilever static scheme.

The unique character of the object results from the fact that it was made as a sheet pile wall of wooden piles driven into the bottom of the shipping channel - the estuary of the Świna River to the Baltic Sea. The age of the structure was estimated at about 60 years.

In the case of a retaining construction, it is important to maintain the serviceability limit state, i.e. limit the horizontal displacement of the cup of the retaining wall.

Displacements of the wall cup depend on the applied loads and strength parameters of the wooden palisade. Impacts result from earth pressure and hydrostatic pressure. Hydrostatic pressure is variable due to changes in the water level in the channel, which are up to about $1.0 \mathrm{~m}$. Fluctuations in the water level cause differentiation of wood parameters along the

\footnotetext{
* Corresponding author: marek.wyjadlowski@pwr.edu.pl
} 
length of the retaining wall. Three zones along the pile can be distinguished here: the part above the water table, the part in the fluctuation zone of the water level and the part permanently immersed in watered ground.

The estimation of displacements of a retaining structure therefore requires determining the range of these zones and the strength parameters of wood.

The work includes testing wood samples obtained from the water level fluctuation zone, exposed to biological corrosion and permanent watering zones. The research aims to determine the environmental impact on the parameters of the wooden structure and its durability.

\section{Testing methods description}

There are different methods for testing timber structures: destructive, semi-destructive (SDT) and non-destructive or quasi on-destructive (NDT).

To obtain detailed data on the physical and mechanical properties of wood both nondestructive and destructive tests should be conducted. Correlation of the results of both these tests provides comprehensive information, which can be helpful in the static analysis of timber construction element or the whole structure.

Nevertheless, non-destructive methods allows to gain some information such as properties of existing construction, estimate the technical condition of the structure or can help in designing reinforcement that may be required. NDT methods also allow to indicate internal damages in the construction material, which are often very difficult to detect.

In this case study two of the non-destructive testing methods were used: ultrasonic and stress wave techniques. Research is still in progress and after more tests more information about the material will be gathered. In this paper current status of research regarding the ultrasonic and stress wave techniques is presented.

\subsection{Idea of acoustic testing methods}

Acoustic testing methods such as ultrasonic and stress wave techniques enable assessment of the physical and mechanical properties of wood based on analysis of the velocity of waves propagated in the tested material. These methods can be used to estimate selected mechanical parameters of material (among others the modulus of elasticity) or to detect internal discontinuities in material.

The basic parameter used in non-destructive methods is the velocity of the wave propagation $(V)$, which is defined by the formula:

$$
V=L / T
$$

where $L$ - distance covered by the wave (between two points of measurement), $T$ - time needed to cover the distance.

Knowing the velocity of propagation and the wood density $(\rho)$, it is possible to determine the dynamic modulus of elasticity $\left(M O E_{d y n}\right)$, which is correlated to the static modulus of elasticity $\left(M O E_{s t a t}\right)$. The dynamic modulus of elasticity $\left(M O E_{d y n}\right)$ can be calculated according to the formula:

$$
M O E_{d y n}=V^{2} \cdot \rho,
$$

where $V$ - velocity of the wave propagation, $\rho$ - density of wood.

The velocity of propagation of the ultrasonic wave depends on the structure of the material.

In wood it depends on directions to the grain (perpendicular or longitudinal). 
According to [1], for wood without defects and flaws the velocity of the ultrasonic wave in the direction perpendicular to the grain ranges between $1000-1500 \mathrm{~m} / \mathrm{s}$ and in the direction longitudinal to the grain between $3500-5000 \mathrm{~m} / \mathrm{s}$. Different velocities may indicate internal discontinuities of wood. According to [1], the velocity of stress wave for healthy wood in the perpendicular direction ranges between $1100-1700 \mathrm{~m} / \mathrm{s}$ and in the longitudinal direction between $3300-5900 \mathrm{~m} / \mathrm{s}$.

The lower velocity perpendicular to the wood fibres results from the internal structure of the material (the wave encounters more cell walls along the way, the crossing of which increases the wave transition time, whereas in parallel direction there are fewer or no barriers, hence the velocity is higher).

\subsection{Description of acoustic methods and devices used in these methods}

There are a few devices used in ultrasonic or stress wave testing methods. In this case study two of them were used and the results are reported in Point 3.

The Fakopp Microsecond Timer device (Fig. 1) uses the stress wave technique. In this method the wave in the material is generated by tapping it with a dedicated hammer. The device measures the time of stress wave propagation between two transducers. To ensure the proper connection between the heads and the sample using some special conducting gel is necessary.

In the ultrasonic wave method there are two ways of registering the wave propagated through the material: direct and indirect method. In the direct method the time of transmitting signal between the receiving head and the transmitting head is measured. The heads are placed on the opposite planes of the sample tested. In the indirect method (echo method) both heads are placed on the same plane of the sample and register the reflected signal. Produced by the Swiss company CBS-CBT Sylvatest Trio portable device (Fig. 1) measures the time needed for the ultrasonic wave to cover a specified distance between two measuring points in the tested sample where the heads are placed. The heads of the device: transmitting and receiving are piezoelectric transducers. To conduct the test, tips of both heads must be placed in specially prepared holes (diameter of $5 \mathrm{~mm}$ and depth of $10 \mathrm{~mm}$ ). In this case no gel is needed. It is to be noticed that the results from Sylvatest Trio device can be influenced by actions inducing other mechanical waves close to testing location due to extremely high sensitivity of the device. Also material's moisture and any internal stresses may affect the results of research significantly.

In order to obtain comprehensive results in both methods, it is recommended to run a large number of measurements in different directions.

a)

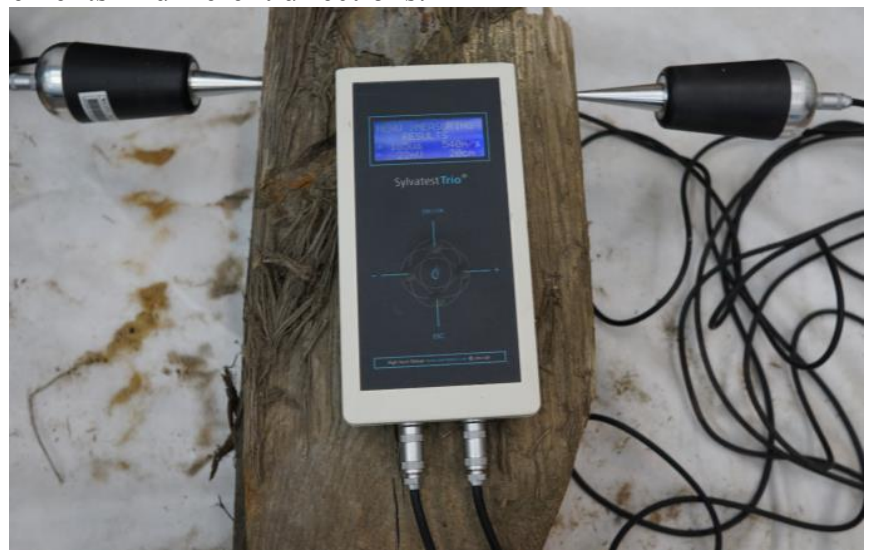


b)

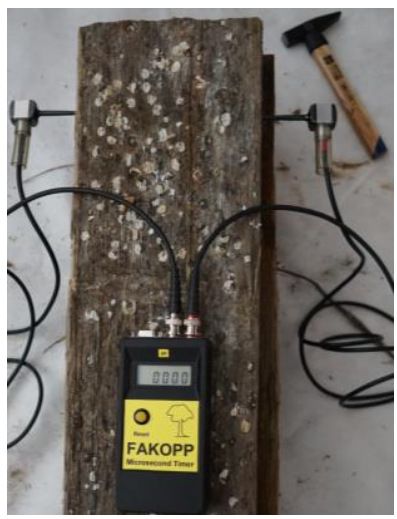

Fig. 1. a) Sylvatest Trio device, b) Fakopp Microsecond Timer device.

\subsection{Correlation between physical and mechanical properties of wood and parameters from acoustic methods}

A number of research using these two methods, attempts to assess their effectiveness and find correlation between physical and mechanical properties of wood and parameters from acoustic testing methods were undertaken (among others [2, 3, 4, 5, 6, 7, 8]).

Due to the research a strong correlation between the dynamic modulus of elasticity $\left(M O E_{d y n}\right)$ and the static modulus of elasticity $\left(M O E_{\text {stat }}\right)$ can be observed. According to [8], for the sound wood free of flaws the determination coefficient for static and dynamic modulus of elasticity is 0,96 . Also several tests evaluating the efficiency of Fakopp Microsecond Timer device and comparing it to Sylvatest Trio device were conducted. These test confirmed effectiveness of using both devices with good results $[7,8]$ and confirmed correlation between the values of $M O E_{d y n}$ and the modulus of elasticity [6].

In different research the reduction of the ultrasonic wave velocity and the stress wave velocity was analysed. According to [10], reduction of the velocity level by $30 \%$ may correspond to $50 \%$ decrease of load-bearing capacity and reduction of velocity level by more than $50 \%$ may indicate considerable damage and significant loss of load-bearing capacity. According to [10], reduction of the velocity of the stress wave propagated between two transducers $\left(\Delta V_{r e l}\right)$, which can be calculated by the formula 3 , describes the material degradation level.

$$
\Delta V_{\text {rel }}=\left[\left(V_{\text {ref }}-V_{\text {mes }}\right) / V_{\text {ref }}\right] \cdot 100 \%,
$$

where $\Delta V_{\text {rel }}$ - relative velocity decrease, $V_{\text {ref }}$ - reference velocity, $V_{m e s}-$ measured velocity. The relation between the relative velocity decrease and the range of destruction due to [Fakopp] is presented in Table 1.

Table 1. Relation between the relative velocity decrease and the destruction range.

\begin{tabular}{|c|c|}
\hline Relative velocity decrease in \% & Destruction range in \% \\
\hline $0-10$ & No destruction \\
\hline $10-20$ & 10 \\
\hline $20-30$ & 20 \\
\hline $30-40$ & 30 \\
\hline $40-50$ & 40 \\
\hline$\geq 50$ & $\geq 50$ \\
\hline
\end{tabular}




\section{Experimental testing}

\subsection{Tested elements}

The Sylvatest Trio and Fakopp Microsecond Timer devices, presented in Point 2, were used for non-destructive tests on timber elements from construction of the port quay. Three elements of the structure (Fig. 2 - 4) were taken from the construction and transported to the laboratory of Wroclaw University of Technology to be tested. Besides acoustic tests, the moisture content was measured using a FMW moisture meter (resistance type with a hammer probe). Elements were tested two times: every time with different moisture content to examine how this can affect the velocity of wave propagation.
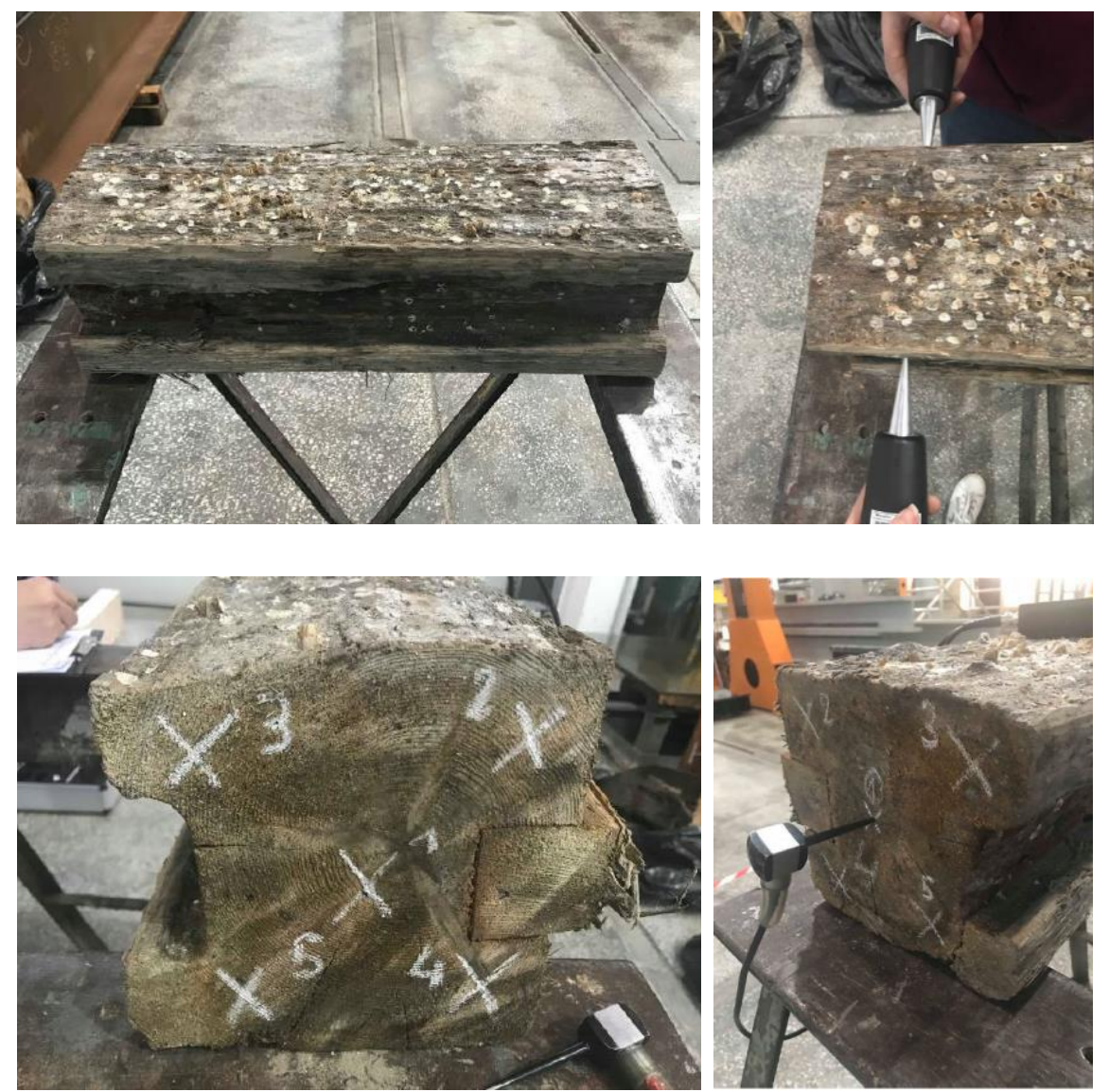

Fig. 2. Element nr 1 (top left), measure points location (bottom left), Sylvatest Trio measuring (top right), Fakopp Microsecond Timer measuring (bottom right). 

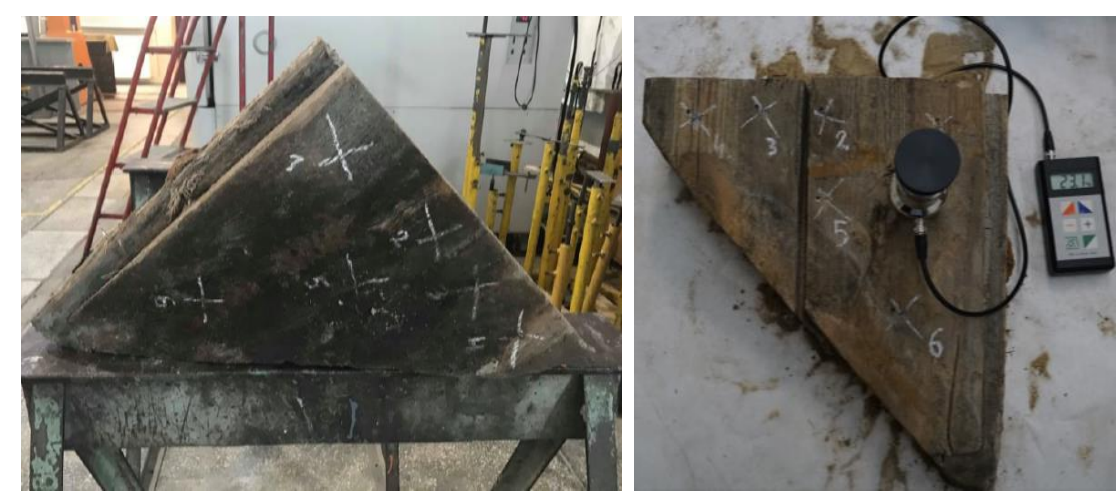

Fig. 3. Element nr 2 with measure points location (left), moisture content measuring (right).
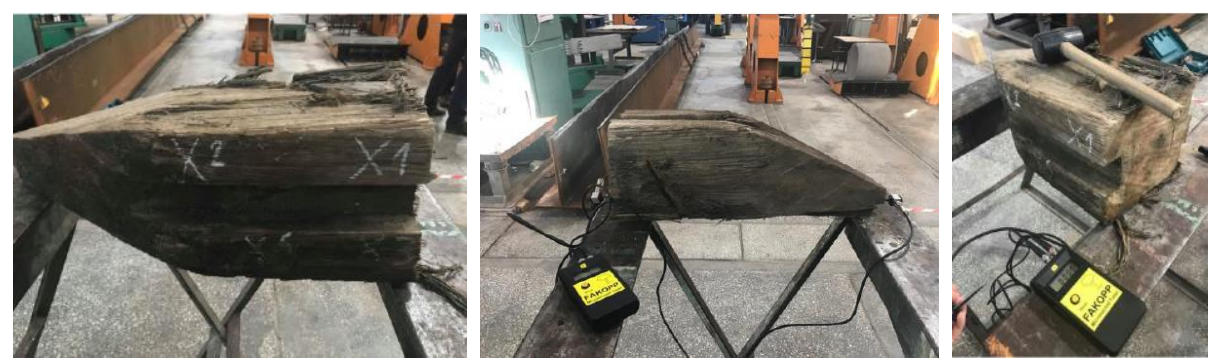

Fig. 4. Element nr 3 with measure points location (left), Fakopp Microsecond Timer measuring (middle and right).

\subsection{Research results}

During research the time of both the stress wave and the ultrasonic wave propagation and the distance between measuring points were measured and the velocity of the wave propagation was calculated for both directions to the grain (parallel and perpendicular). In the next step, assuming the density of wood elements for $420 \mathrm{~kg} / \mathrm{m}^{3}$ (research still in progress, the density will be examined), the dynamic modulus of elasticity was calculated. The results are presented in Tables 2, 3 .

Table 2. Results from Fakopp Microsecond Timer test (velocity of the stress wave propagation and the dynamic modulus of elasticity).

\begin{tabular}{|c|c|c|c|c|c|}
\hline \multirow{2}{*}{ Element } & \multirow{2}{*}{$\begin{array}{c}\text { Direction } \\
\text { to the grain }\end{array}$} & \multicolumn{2}{|c|}{ Moisture content: about 30\% } & \multicolumn{2}{c|}{ Moisture content: $24-28 \%$} \\
\cline { 3 - 6 } & & $\begin{array}{c}V \\
{[\mathrm{~m} / \mathrm{s}]}\end{array}$ & $\begin{array}{c}\text { MOEdyn } \\
{[\mathrm{GPa}]}\end{array}$ & $\begin{array}{c}V \\
{[\mathrm{~m} / \mathrm{s}]}\end{array}$ & $\begin{array}{c}\text { MOEdyn } \\
{[\mathrm{GPa}]}\end{array}$ \\
\hline \multirow{3}{*}{1} & $\|$ & 4872,8 & 9,97 & 5376,6 & 12,14 \\
\cline { 2 - 6 } & $\perp$ & 1153,7 & 0,56 & 1439,9 & 0,87 \\
\hline \multirow{2}{*}{2} & $\|$ & - & - & - & - \\
\cline { 2 - 6 } & $\perp$ & 1218,5 & 0,62 & 1372,8 & 0,79 \\
\hline \multirow{3}{*}{3} & $\|$ & - & - & - & - \\
\cline { 2 - 6 } & $\perp$ & 1406,1 & 0,83 & 1447,7 & 0,88 \\
\hline
\end{tabular}


Table 3. Results from Sylvatest Trio test (velocity of the ultrasonic wave propagation and the dynamic modulus of elasticity) .

\begin{tabular}{|c|c|c|c|c|c|}
\hline \multirow{2}{*}{ Element } & \multirow{2}{*}{$\begin{array}{c}\text { Direction } \\
\text { to the grain }\end{array}$} & \multicolumn{2}{|c|}{ Moisture content: about 30\% } & \multicolumn{2}{c|}{ Moisture content: $24-28 \%$} \\
\cline { 3 - 6 } & & $\begin{array}{c}V \\
{[\mathrm{~m} / \mathrm{s}]}\end{array}$ & $\begin{array}{c}M O E_{d y n} \\
{[\mathrm{GPa}]}\end{array}$ & $\begin{array}{c}V \\
{[\mathrm{~m} / \mathrm{s}]}\end{array}$ & $\begin{array}{c}\text { MOEdyn } \\
{[\mathrm{GPa}]}\end{array}$ \\
\hline \multirow{3}{*}{1} & $\|$ & 5128,9 & 11,05 & 5855,4 & 14,40 \\
\cline { 2 - 6 } & $\perp$ & 1118,4 & 0,53 & 1311,5 & 0,72 \\
\hline \multirow{2}{*}{2} & $\|$ & - & - & - & - \\
\cline { 2 - 6 } & $\perp$ & 1030,24 & 0,45 & 1311,4 & 0,72 \\
\hline \multirow{3}{*}{3} & $\|$ & - & - & - & - \\
\cline { 2 - 6 } & $\perp$ & 1199,8 & 0,60 & 1251,1 & 0,66 \\
\hline
\end{tabular}

The correlation of the dynamic moduli of elasticity was calculated for measurements directions parallel and perpendicular to the grain. The results are shown in Fig. 5.

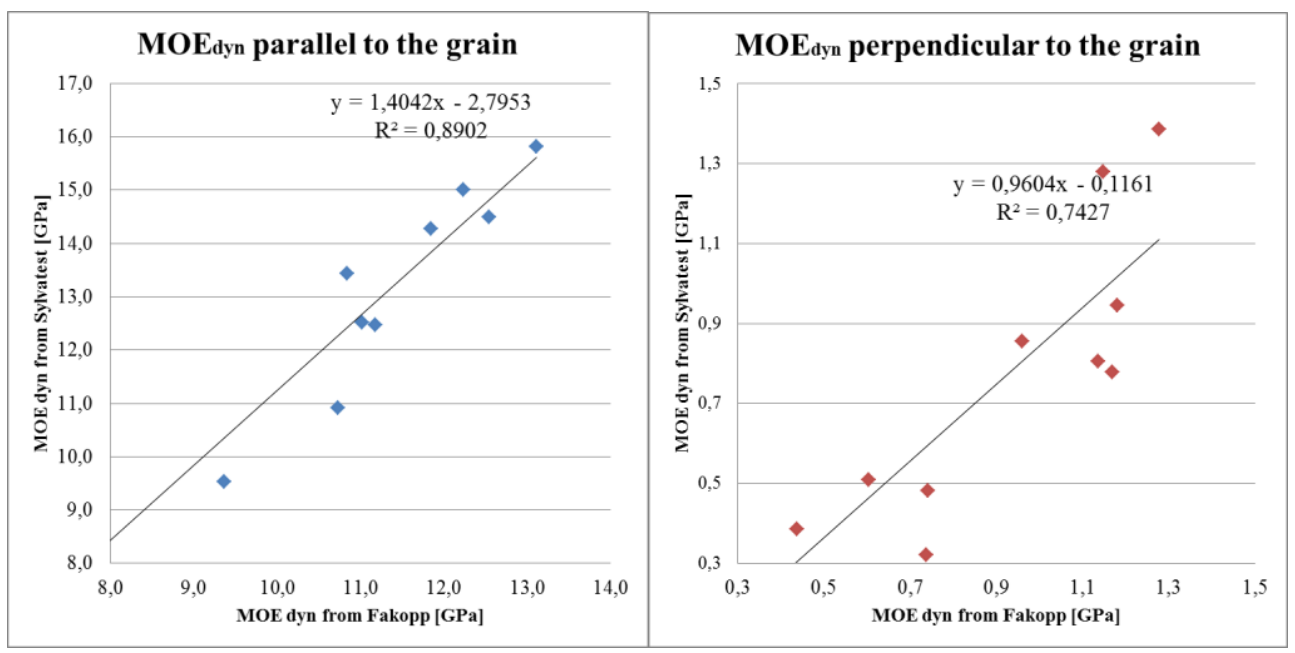

Fig. 5.The correlation of the dynamic modulus of elasticity determined in the stress wave method (Fakopp) and the ultrasonic wave method (Sylvatest) for both directions parallel and perpendicular .

\subsection{Discussion}

Analysing values of the velocities of the wave propagation measured in both methods, it can be seen that above mentioned values differ from each other regarding different moisture contents. The velocities for the elements with higher moisture level (about 30\%) are lower than the velocities for elements with lower moisture level. Nevertheless, the values obtained by both methods correspond to the values from literature $[9,10,11]$.

In turn, considering values of the dynamic modulus of elasticity, the lower moisture content, the higher values are obtained.

What can be considered as surprising, no significant difference in the estimated parameters values for each elements can be observed. Regardless to the original elements 
location in the structure (under water, in the ground or flooded and unflooded) measured values are similar.

It is also worth to mention that the results from the ultrasonic method are more accurate among tested elements than the results from wave stress method.

\section{Conclusions}

The results presented below can lead to the conclusion that both methods: the stress wave by Fakopp Microsecond Timer device and the ultrasonic wave by Sylvatest Trio correspond with each other and result in similar outcomes. The coefficient of determination is 0,89 for $M O E_{d y n}$ parallel to the grain and 0,74 for $M O E_{d y n}$ perpendicular to the grain, which can be considered sufficient. It can be concluded that applying these two methods together can allow to obtain satisfying results regarding estimation of the dynamic modulus of elasticity.

However, in order to obtain more detailed and comprehensive information on the elements properties, more research should be conducted. They will allow to estimate more parameters, such as the static modulus of elasticity, which may be used in the global structural analysis. It is necessary to run a higher number of tests covering the whole element to obtain more detailed results. Further investigation of the elements are planned.

\section{References}

1. R. Ross, B. Brashaw, X. Wang, R. White, R. Pellerin, Wood and Timber: Condition Assessment Manual, Madison, WI, Forest Products Society (2004)

2. T. Lechner, T. Nowak R. Klinger, In situ assessment of the timber floor structure of the the Skansen Lejonet fortification, Sweden, Construction and Building Materials 58, 8593 (2014)

3. M. Garcia, J. Seco, E. Prieto, Improving the prediction of strength and rigidity of structural timber by combining ultrasound techniques with visual grading parameters, Materiales de Construcción 57(288), 49-59 (2007)

4. P. Lourenco, A. Feio, J. Machado, Chestnut wood in compression perpendicular to the grain: Non-destructive correlations for test results in new and old wood, Construction and Building Materials 21(8), 1617-1627 (2007)

5. T. Ilharco, T. Lechner, T. Nowak, Assessment of timber floors by means of nondestructive testing methods, Construction and Building Materials, http://dx.doi.org/10.1016/j.conbuildmat.2015.05.133 (2015)

6. T. Nowak, K. Hamrol-Bielecka, J. Jasieńko, Experimental testing of glued laminated timber members using ultrasonic and stress wave techniques, Proc. of the International Conference on Structural Health Assessment of Timber Structures - SHATIS'15, Wroclaw, 9-11 September, 523-533 (2015)

7. M. Esteban, F. Arriaga, G. Iniguez, I. Bobadilla, Structural assessment and reinforcement of ancient timber trusses, Proc. of the International Conference on Structures \& Architecture, 21-23 July, Guimarães, Portugal (2010)

8. G. Iniguez, R. Martinez, I. Bobadilla, F. Arriaga, M. Esteban, Mechanical properties assessment of structural coniferous timber by means of parallel and perpendicular to the grain wave velocity, Proc. of the 16th International Symposium on Nondestructive Testing of Wood, Beijing, China (2009)

9. B. Kasal, T. Tannert (eds), In Situ Asessment of Structural Timber, New York, Springer (2010) 
10. U. Dachermann, K. Crews, B. Kasal, J. Li, M. Riggio, F. Rinn, T. Tannert, In situ assessment of structural timber using stress-wave measurements, Materials and Structures 47(5), 787-803 (2014)

11. Fakopp Enterprise Microsecond Timer, http://www. fakopp.com/site/microsecondtimer (2012) 